\title{
Rehabilitación de la marcha en el paciente con ictus Revisión bibliográfica
}

\author{
MANUEL José PinAZO Llobet \\ manuelpi94@gmail.com \\ María Arantzazu Ruescas nicolau \\ arancha.ruescas@uv.es
}

\section{Resumen}

Introducción: La rehabilitación de la marcha es el objetivo principal de la fisioterapia en el ictus. Dos de los tratamientos más utilizados son el entrenamiento convencional de la marcha (ECM) y el entrenamiento de la marcha sobre cinta rodante con soporte de peso (EMSP). El objetivo principal de esta revisión ha sido realizar una revisión bibliográfica de la literatura científica, sobre la comparación entre el EMSP y el ECM en función de los resultados de las variables velocidad de la marcha, capacidad funcional y equilibrio. Y secundariamente, se ha pretendido evidenciar su eficacia en función de la cronicidad del ictus. Metodología: Se realizó una búsqueda bibliográfica en siete bases de datos EMBASE, Scopus, Literatura Latinoamericana y del Caribe en Ciencias de la Salud (LILACS), Web of Science (ISI), Physiotherapy Evidence Database (PEDro) y Cochrane, limitándola a estudios experimentales, prospectivos, aleatorizados y controlados, publicados entre enero de 2010 y diciembre de 2015 en lengua inglesa o española. Resultados: Se incluyeron 11 artículos. Nuestros resultados parecen indicar una tendencia hacia la mejoría superior en el tratamiento EMSP. No obstante, no existen diferencias estadísticamente significativas que lo abalen. Conclusión: No hemos encontrado evidencia para confirmar que el EMSP es un tratamiento más eficaz que el ECM, aunque clínicamente parece haber una mayor mejoría con el EMSP. La respuesta al tratamiento varía en función de la cronicidad. De modo que, los pacientes agudos tienen una mayor progresión en su capacidad de deambular, mientras que los crónicos mantienen la funcionalidad de la marcha.

Palabras clave: Ictus, rehabilitación de la marcha, soporte de peso, entrenamiento sobre el suelo, tapiz rodante.

\section{Abstract}

Introduction: Gait rehabilitation is the main objective of the physiotherapy in the stroke. Two of the most used treatments in conventional walking training (ECM) and treadmill training with bodyweight support (EMSP). The main objective of this review has been to analize the scientific literature on the comparison between EMSP and ECM in the function of the results of variables gait velocity, functional capacity and balance. Secondarily, it has been tried to show its efficacy in the function of the chronicity of the stroke. Methodology: A bibliographic search was conducted in seven databases EMBASE, Scopus, 
Latin American and Caribbean Literature in Health Sciences (LILACS), Web of Science (ISI), Physiotherapy Evidence Database (PEDro) and Cochrane, limiting it to experimental, prospective, randomized and controlled studies, published between January 2010 and December 2015 in English or Spanish. Results: Eleven articles were included. Our results seem to indicate a trend toward superior improvement in EMSP treatment. However, there are no statistically significant differences between the two treatments. Conclusion: We found no evidence to confirm that PFME is a more effective treatment than ECM, although clinically there seems to be a greater improvement with PFME. The response to treatment depends on the chronicity of the stroke. Acute patients have a greater progression in their ability to ambulate, while the chronic ones maintain gait functionality.

Keywords: gait rehabilitation, treadmill, bodyweight support, overground training.

\section{Introducción}

La incidencia del ictus en España es de 128 casos por cada 100.000 habitantes. En cuanto a la prevalencia, se estima que es de un $6,4 \%$, siendo mayor en hombres $(7,3 \%)$ que en mujeres $(5,6 \%)$.

En España, la mortalidad ha disminuido y la morbilidad ha aumentado (Brea et al., 2013). Seis meses después del ictus, el $26,1 \%$ de los pacientes han fallecido y el $44 \%$ de los supervivientes quedan con una dependencia funcional (Heras et al., 2004). Se estima que el coste socioeconómico de esta enfermedad supone entre el $3 \%$ y el $4 \%$ del gasto sanitario total de los países con rentas elevades (Mar et al., 2013) alrededor de 6.000 millones de euros anuales a nivel español (Jorgensen et al., 2008).

El objetivo principal de la fisioterapia en el ictus es la recuperación de la funcionalidad del paciente. Para ello, se debe centrar el proceso de rehabilitación en la reeducación de la marcha.

Para la reeducación de la marcha, el enfoque terapéutico con mayor repercusión, ha sido el reaprendizaje motor orientado a tareas. Dos de las técnicas fundadas bajo este enfoque terapéutico que más se utilizan en la actualidad son el entrenamiento de la marcha sobre el suelo $(E C M)$ y entrenamiento de la marcha sobre una cinta rodante con soporte del peso corporal del paciente (EMSP).

En la actualidad se ha generalizado la utilización de la técnica EMSP. Este tipo de tratamiento consiste en utilizar un sistema de suspensión unido a un arnés para soportar un porcentaje del peso del paciente mientras éste camina sobre una cinta de marcha. El sistema permite ejercitar la marcha de forma rítmica y repetitiva. Este tipo de tratamiento se presenta como más completo ya que, reduce la carga que debe desplazar el paciente durante la marcha y permite dar un numero de pasos por sesión mayor que el ECM (Hesse et al., 2003).

Las revisiones existentes hasta la fecha, no compararon de forma exclusiva los dos tipos de entrenamiento de la marcha, ECM y EMSP, que en la presente revisión se pretenden comparar. Además, en la práctica clínica los principales indicadores de los cambios en la funcionalidad de la marcha en los pacientes con ictus son la velocidad, la capacidad funcional y el equilibrio (Brandstater et al., 1983). La velocidad y la capacidad funcional sí han sido estudiadas, sin embargo, a pesar de la vital importancia del equilibrio en la adquisición de una marcha segura y funcional, ninguna revisión hasta el momento ha evaluado la eficacia de ambos tratamientos sobre esta variable. 
Por lo anteriormente expuesto, la presente revisión parte de la hipótesis de que el método EMSP es una forma de reeducación de la marcha más eficaz que el método ECM para mejorarla velocidad de la marcha, capacidad funcional y equilibrio en personas con secuelas de ictus.

Así pues, el presente trabajo tiene como objetivo principal realizar una revisión bibliográfica de la literatura científica publicada entre 2010 y 2015 sobre la eficacia del EMSP versus el ECM en la reeducación de la marcha de pacientes con secuelas de ictus. Concretamente se pretende analizar su eficacia a nivel de velocidad de marcha, capacidad funcional y equilibrio. Secundariamente, se pretende evidenciar la eficacia de estos tratamientos en función de la cronicidad del ictus.

\section{Método}

\section{Estrategia de búsqueda}

Se llevó a cabo una búsqueda sistemática en las siguientes bases de datos electrónicas: EMBASE, Scopus, Literatura Latinoamericana y del Caribe en Ciencias de la Salud (LILACS), Web of Science (ISI), Physiotherapy Evidence Database (PEDro) y Cochrane.

Para establecer los términos de búsqueda se empleó el tesauro Descriptores en Ciencias de la Salud (DeCS) y el propio de cada base de datos. Una vez definidos, se combinaron los términos de búsqueda relacionados con la rehabilitación de la marcha (gaitrehabilitation, treadmill, bodyweight, exercicetherapy, musclestrenght, balance, gaitspeed y overground training) con los términos que representaban a la población estudiada (stroke) a través del uso de los operadores booleanos AND y OR.

La búsqueda se limitó a estudios experimentales, prospectivos, aleatorizados y controlados, publicados entre enero de 2008 y diciembre de 2015 en lengua inglesa o española.

\section{Criterios de inclusión y exclusión}

Se incluyeron aquellos ensayos clínicos que estaban disponibles a texto completo y que estudiaban a sujetos con secuelas de ictus tanto en fase subaguda como crónica, y donde se comparara el ECM y el EMSP. Se seleccionaron las publicaciones que valoraron el efecto del entrenamiento aplicado en ambos grupos sobre, al menos, una de las siguientes variables: equilibrio estático, velocidad de la marcha y capacidad funcional.

Se excluyeron aquellos trabajos en los que la muestra no estuviera compuesta por personas con secuelas de ictus, aquellos que no contemplaban ninguna de las variables anteriormente mencionadas, aquellos cuyos pacientes pertenecientes al grupo de entrenamiento con soporte de peso fueran asistidos por robots y aquellos en los que el grupo control no realizara ningún tipo de tratamiento o bien el tratamiento que recibían no incluía la marcha sobre el suelo.

\section{Selección de los artículos}

Tras realizar la búsqueda bibliográfica, se procedió a la lectura del título, y del resumen, si era necesario. A continuación, cuando fue posible localizar el texto completo, se procedió a la lectura integra de éste. Por último, si el trabajo cumplía los criterios de inclusión, se realizó el proceso de extracción de datos. 


\section{Evaluación del riesgo de sesgo}

La evaluación del riesgo de sesgo de los estudios seleccionados se realizó mediante las escalas de Jadad (Jadad et al., 1996) y PEDro (De Morton et al., 2009). Ningún estudio fue excluido del análisis final tras la evaluación del riesgo de sesgo.

\section{Extracción de datos}

La extracción de datos se hizo en base a un formulario, previamente definido, donde se recogía información correspondiente a la población estudiada, variables analizadas, intervenciones aplicadas, resultados obtenidos y riesgo de sesgo.

\section{Resultados}

\section{Selección de estudios}

A partir de los resultados obtenidos en cada base de datos tras la aplicación de la estrategia de búsqueda, se identificaron un total de 221 publicaciones, 37 de las cuales estaban duplicadas (Figura 1). Después de realizar una revisión del título y resumen de cada publicación, se excluyeron 169 estudios por no cumplir los criterios de inclusión: población inadecuada $(n=19)$, intervención inapropiada $(n=90)$, diseño inadecuado $(n=29)$ y variables inapropiadas $(n=31)$. De modo que, finalmente, 15 estudios fueron seleccionados para la evaluación del texto completo. De estos, se excluyeron 4 artículos por imposibilidad de recuperación del texto completo $(n=2)$, intervención inadecuada $(n=1)$ o variables inadecuadas $(n=1)$. Finalmente 11 estudios fueron incluidos para su revisión.

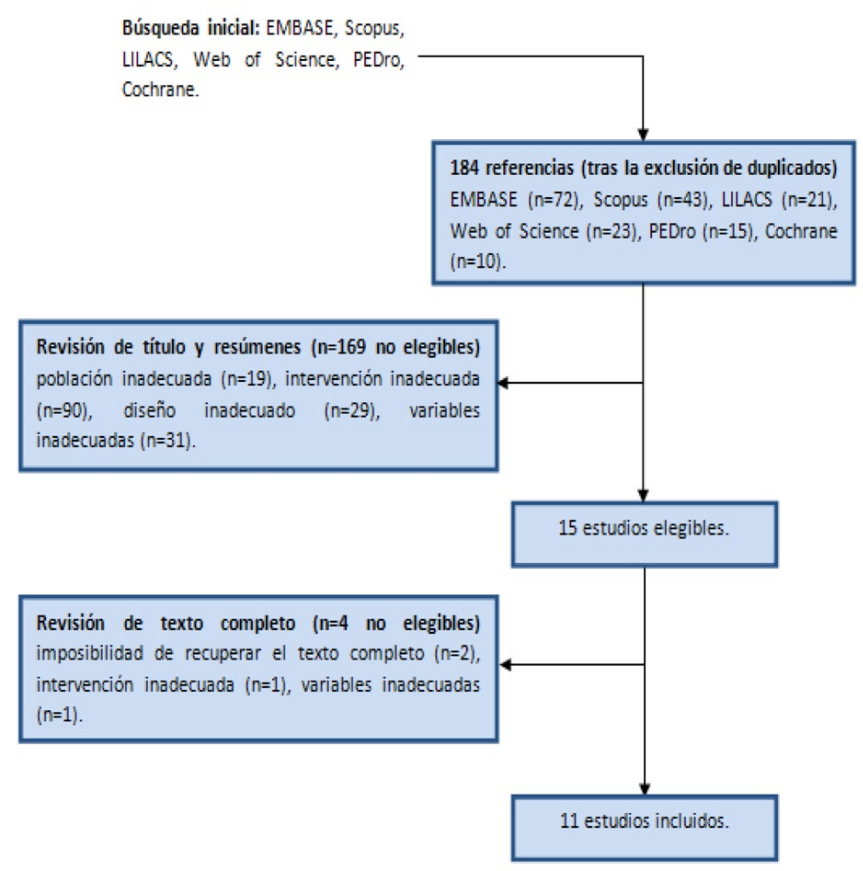

Figura 1. Diagrama de flujo de la selección de artículos 
Riesgo de sesgo en los estudios incluidos

Atendiendo a la puntuación mínima establecida por cada escala para determinar que un estudio tiene una calidad metodológica aceptable ( $\geq 7$ para la escala PEDro, $\geq 3$ para la escala Jadad), cinco investigaciones superaron ambas puntuaciones (Combs-Miller et al., 2014; De Paul et al., 2015; Duncan et al., 2011; MacKay-Lyons et al., 2013; Middleton et al., 2014), otras dos (Høyery et al., 2012; Mao et al., 2015;) superaron la puntuación de la escala PEDro pero no la de la escala Jadad y una (Takami \& Wakayama, 2010) superó la de la escala Jadad, pero no la de la escala PEDro. Así pues, de los once artículos incluidos en esta revisión, solo tres (Middleton et al., 2014; Ribeiro et al., 2013; Park et al., 2015) no presentaron una calidad metodológica aceptable.

\section{Efecto de las intervenciones}

a) Velocidad de la marcha

Los 11 estudios incluidos en esta revisión analizaron la velocidad de la marcha, 9 midieron esta variable con el test 10 metros marcha, uno (Middleton et al., 2014) con el test 3 metros marcha y otro (De Paul et al., 2015) con el test 5 metros marcha.

Los resultados de los 11 estudios muestran que el grupo EMSP tiende a tener una mejoría clínica mayor, ya que solo en dos estudios (Combs-Miller et al., 2014; Park et al., 2015) ocurrió lo contrario. No obstante, solo se encontraron diferencias estadísticamente significativas en tres trabajos (Combs-Miller et al., 2014; Mao et al., 2015; Langhammer \& Stanghelle, 2010).

\section{b) Capacidad funcional}

En siete estudios (Combs-Miller et al., 2014; De Paul et al., 2015; Duncan et al., 2011; Høyer et al., 2012; Langhammer \& Stanghelle, 2010; MacKay-Lyons et al., 2013; Middleton et al., 2014;) se evaluó la capacidad funcional mediante el test 6 minutos marcha. Aunque en los siete estudios la mejoría fue mayor en el grupo EMSP, solo en el trabajo de MacKay-Lyons et al., (2013) las diferencias fueron estadísticamente significativas a favor del grupo EMSP.

c) Equilibrio estático

Cuatro de los once estudios incluidos en esta revisión valoraron el equilibrio (Duncan et al., 2011; MacKay-Lyons et al., 2013; Middleton et al., 2014; Takami \& Wakayama, 2010) utilizando la escala de Berg. Ningún estudio encontró diferencias estadísticamente significativas, aunque en tres de los cuatro estudios el grupo ECM obtuvo mejor puntuación que el grupo EMSP y, solo en el trabajo de Takami y Wakayama (2010) ocurrió lo contrario.

\section{Discusión y conclusiones}

Los resultados del presente trabajo no han sido concluyentes. Por lo tanto, a la espera de futuras investigaciones más esclarecedoras, no podemos afirmar que el EMSP sea una técnica más eficaz que el ECM. Respecto a la cronicidad del ictus, como cabía esperar, las 
mejorías son superiores en los pacientes agudos en ambos tratamientos. No obstante, los pacientes crónicos también mejoran, en los dos tratamientos, de modo que ambos tienen una función de mantenimiento de la capacidad de marcha en este grupo poblacional.

La ausencia de evidencia científica a favor de la técnica EMSP, sugiere que las decisiones de los terapeutas deberían tomarse considerando las preferencias del paciente, así como el equipamiento y personal disponible. El ECM es un tratamiento más económico, ya que requiere un equipamiento menor, pero es cierto que los pacientes que reciben este tratamiento necesitan un número de sesiones mayor para igualar los resultados del grupo EMSP. Si traducimos este dato al gasto sanitario total, la balanza económica podría igualarse, de modo que descartar el EMSP por razones económicas no sería un motivo de peso suficiente, aunque sí lo podrían ser las instalaciones y el personal del que se disponga.

\section{Limitaciones y futuras líneas de investigación}

Esta revisión presenta varias limitaciones. Primero, en cuanto a la realización de este trabajo, haber limitado la búsqueda a textos disponible a texto completo, redactado en inglés o español y publicado en los últimos 5 años, puede haber sido un factor determinante que ha limitado el acceso a la totalidad de la evidencia disponible.

Por otra parte, respecto a la metodología de los estudios, una debilidad importante de esta revisión ha sido la elevada variabilidad metodológica en la aplicación de las intervenciones. La ausencia de homogeneidad a la hora de aplicar cada tipo de tratamiento, así como la falta de consenso en la dosificación y protocolización de ambas intervenciones, han sido un obstáculo. Otra limitación fue que la mayoría de los estudios incluidos tuvieron un tamaño muestral reducido y esto, posiblemente haya producido un aumento de la dificultad para establecer diferencias estadísticamente significativas entre los dos tratamientos a comparar.

A pesar de esto, la elevada calidad metodológica de la mayoría de los estudios analizados, avala los resultados que en esta revisión se presentan.

Los resultados de la presente revisión han evidenciado la necesidad de futuros ensayos clínicos en este campo que limiten ambas intervenciones únicamente a los dos tipos de tratamiento, sin introducir otro tipo de terapias como el entrenamiento de la fuerza y sobretodo el equilibrio, ya que esto puede alterarlos resultados e impedir una correcta comparación entre tratamientos. También sería conveniente estudiar las características más apropiadas para la dosificación de los parámetros del entrenamiento (intensidad, duración, frecuencia). Asimismo, consideramos importante realizar futuros ensayos clínicos con un mayor tamaño muestral y donde se haga un seguimiento a largo plazo para poder observar el mantenimiento de los beneficios de ambas intervenciones.

\section{Conclusiones}

A partir de los resultados obtenidos en la presente revisión podemos concluir que:

1) No existe evidencia que afirme que, para reeducar la marcha en personas con secuelas de ictus, el entrenamiento con soporte de peso sea más eficaz que el entrenamiento convencional.

2) Parece que el entrenamiento de suspensión tiende a producir una mejoría en la velocidad de la marcha, clínicamente superior, al entrenamiento convencional. 
3) El entrenamiento con soporte de peso, parece ser más eficaz que el entrenamiento de la marcha sobre el suelo para la mejora de la capacidad funcional.

4) Parece que ambos tratamientos son útiles para la mejoría del equilibrio, aunque no hemos hallado evidencia para afirmar que un tratamiento es superior al otro.

5) En pacientes agudos parece que hay una tendencia a una recuperación de la capacidad de deambular más rápida con el tratamiento de suspensión. En pacientes con secuelas crónicas de ictus parece que este tratamiento es más eficaz para el mantenimiento de la funcionalidad de la marcha.

\section{Referencias bibliográficas}

Brandstater, M. E., De Bruin, H., Gowland, C. \& Clark, B. M. (1983). Hemiplegic gait: analysis of temporal variables. Archives of Physical Medicine and Rehabilitation, 64(12), 583-587.

Brea, A., Laclaustra, M., Martorell, E. \& Pedragosa, À. (2013). Epidemiología de la enfermedad vascular cerebral en España. Clínica e Investigación en Arteriosclerosis, 25(5), 211-217.

Combs-Miller, S. A., Kalpathi Parameswaran, A., Colburn, D., Ertel, T., Harmeyer, A... Schmid, A. A. (2014). Body weight-supported treadmill training vs. overground walking training for persons with chronic stroke: a pilot randomized controlled trial. Clinical Rehabilitation, 28(9), 873-884.

De Morton, N. A. (2009). The PEDro scale is a valid measure of the methodological quality of clinical trials: a demographic study. Australian Journal of Physiotherapy, 55(2), 129-133.

De Paul, V. G., Wishart, L. R., Richardson, J., Thabane, L., Ma, J. \& Lee, T. D. (2015). Varied Overground Walking Training Versus Body-Weight-Supported Treadmill Training in Adults Within 1 Year of Stroke A Randomized Controlled Trial. Neurorehabilitation and Neural Repair, 29(4), 329-340.

Duncan, P. W., Sullivan, K. J., Behrman, A. L., Azen, S. P., Wu, S. S., Nadeau, S. E...Hayden, S.K. (2011). Body-weight-supported treadmill rehabilitation after stroke. New England Journal of Medicine, 364(21), 2026-2036.

Heras, M. A., de la Cámara-González, C., Tacsir, A. \& García, J. R. (2004). Informe sobre el impacto social de los enfermos dependientes por ictus: Informe ISEDIC, 2004. Madrid: Egraf.

Hesse, S., Werner, C., Seibel, H., von Frankenberg, S., Kappel, E. M., Kirker, S. \& Käding, M. (2003). Treadmill training with partial body-weight support after total hip arthroplasty: a randomized controlled trial. Archives of Physical medicine and Rehabilitation, 84(12), 1767-1773.

Høyer, E., Jahnsen, R., Stanghelle, J. K. \& Strand, L. I. (2012). Body weight supported treadmill training versus traditional training in patients dependent on walking assistance after stroke: a randomized controlled trial. Disability and Rehabilitation, 34(3), 210-219.

Jadad, A. R., Moore, R. A., Carroll, D., Jenkinson, C., Reynolds, D. J., Gavaghan, D. J. \& McQuay, H. J. (1996). Assessing the quality of reports of randomized clinical trials: is blinding necessary? Controlled Clinical Trials, 17(1), 1-12.

Jorgensen, N., Cabañas, M., Oliva, J., Rejas, J. \& León, T. (2008). Los costes de los cuidados informales asociados a enfermedades neurológicas discapacitantes de alta prevalencia en España. Neurología, 23(1), 29-39.

Langhammer, B. \& Stanghelle, J. K. (2010). Exercise on a treadmill or walking outdoors? A randomized controlled trial comparing effectiveness of two walking exercise programmes late after stroke. Clinical Rehabilitation, 24(1), 46-54. 
MacKay-Lyons, M., McDonald, A., Matheson, J., Eskes, G. \& Klus, M. A. (2013). Dual effects of body-weight supported treadmill training on cardiovascular fitness and walking ability early after stroke: a randomized controlled trial. Neurorehabilitation and Neural Repair, 27(7), 644-653.

Mao, Y. R., Lo, W. L., Lin, Q., Li, L., Xiao, X., Raghavan, P. \& Huang, D. F. (2015). The effect of body weight support treadmill training on gait recovery, proximal lower limb motor pattern, and balance in patients with subacute stroke. BioMedi Research International, 175719.

Mar, J., Álvarez-Sabín, J., Oliva, J., Becerra, V., Casado, M. A., Yébenes, M... MasJuan, J. (2013). Los costes del ictus en España según su etiología. El protocolo del estudio CONOCES. Neurología, 28(6), 332-339.

Middleton, A., Merlo-Rains, A., Peters, D. M., Greene, J. V., Blanck, E. L., Moran, R. \& Fritz, S.L. (2014). Body Weight-Supported Treadmill Training Is No Better Than Overground Training for Individuals with Chronic Stroke: A Randomized Controlled Trial. Topics in stroke rehabilitation, 21(6), 462-476.

Park, B. S., Kim, M. Y., Lee, L. K., Yang, S. M., Lee, W. D., Noh, J. W... Kim, J. (2015). Effects of conventional overground gait training and a gait trainer with partial body weight support on spatiotemporal gait parameters of patients after stroke. Journal of Physical Therapy Science, 27(5), 1603-1607.

Ribeiro, T., Britto, H., Oliveira, D., Silva, E., Galvão, E. \& Lindquist, A. (2013). Effects of treadmill training with partial body weight support and the proprioceptive neuromuscular facilitation method on hemiparetic gait: a randomized controlled study. European Journal of Physical and Rehabilitation Medicine, 49(4), 451-461.

Takami, A. \& Wakayama, S. (2010). Effects of partial body weight support while training acute stroke patients to walk backwards on a treadmill-a controlled clinical trial using randomized allocation. Journal of Physical Therapy Science, 22(2), 177-187. 